\title{
Focal task specific dystonia: a review and update
}

\author{
Christine M. Stahl ${ }^{1} \cdot$ Steven J. Frucht $^{1}$
}

Received: 14 October 2016/Revised: 16 December 2016/Accepted: 19 December 2016/Published online: 30 December 2016 (C) The Author(s) 2016. This article is published with open access at Springerlink.com

\begin{abstract}
In this review, we summarize recent advances in understanding the etiology, risk factors and pathophysiology of focal task specific dystonia (FTSD), movement disorders characterized by abnormal motor activation during the performance of specific, repetitive actions. We focus on two common FTSD, musician's dystonia and writer's cramp. FTSD may pose a threat to the patient's livelihood, and improved therapeutic treatments are needed.
\end{abstract}

Keywords Focal task specific dystonia (FTSD) - Writer's cramp · Musician's dystonia

\section{Introduction}

Dystonias are a diverse "group of movement disorders characterized by sustained or intermittent muscle contractions causing abnormal, often repetitive, movements, postures, or both" [1]. Recently updated consensus opinion classifies dystonias by two axes: clinical characteristics and etiology. The first axis, clinical characteristics, includes the age of onset, affected body region, temporal pattern, and associated neurologic or systemic features [1]. A subclassification includes focal task specific dystonias (FTSD), which are a diverse group of focal dystonias affecting an isolated body part and are triggered, at least initially, by a specific action.

Steven J. Frucht

steven.frucht@mssm.edu

1 Icahn School of Medicine at Mount Sinai, 5 East 98th Street, Box 1138, First Floor, New York, NY 10029, USA
While the term "dystonia" was first used in 1911 by Oppenheim, the clinical phenomenon had been described almost a century earlier in patients with FTSD [2]. In 1830, clerks in the British Civil Service were noted to develop difficulty with writing. After observing these clerks, Sir Charles Bell remarked that he "found the action necessary for writing gone, or the motions so irregular as to make the letters be written zig-zag, whilst the power of strongly moving the arm for fencing remained..." [3]. Later in the 1860s, Samuel Solly labeled this condition "scrivener's palsy" [4]. While "scrivener's palsy" or writer's cramp, as it is now called, is one of the more recognized forms of FTSD, dystonia may affect musicians, typists, hairdressers, painters, shoemakers and tailors [5-7]. Sport-related FTSD have also been described in golfers, pistol shooters and ping-pong players, among others. Because of their association with repetitive, fine motor tasks often linked to one's profession, FTSD have also been referred to as occupational dystonias. Interestingly, until the 1980s FTSD were erroneously interpreted to be psychogenic in origin, often termed "occupational neuroses." In 1982, evidence for an organic etiology emerged from the work of Sheehy and Marsden [8].

In this review, we discuss the phenomenology and epidemiology of two of the most common forms of FTSD, writer's cramp and musician's dystonia. We then discuss emerging findings on the etiology, pathophysiology and treatment of FTSD.

\section{Background}

FTSD typically begins in adulthood with symptom onset in the third to sixth decade. Unlike other adult onset primary focal dystonias, FTSD is more common in men, and it 
usually affects the arm, facial muscles or larynx [9]. Overall prevalence estimates for FTSD in the general population range from 7 to 69 per million [10, 11]. However, prevalence has been estimated to be much higher in selected groups; for instance, some studies have shown as many as $14 \%$ of patients seen at performing arts medical centers have FTSD [12, 13].

FTSD typically presents as an insidious, painless loss of dexterity triggered by performance of a specific, often over-practiced task. Symptoms progress over time to trigger uncontrolled activation of muscle groups, leading to abnormal postures and movements. Early in the disease course, the dystonia typically is triggered only by the performance of a specific task, but over time spreads to involve other tasks, or even spreads to previously unaffected areas of the body. As with other types of dystonias, sensory tricks, or geste antagonistes, may temporarily reduce the dystonic symptoms of FTSD [14].

\section{Writer's cramp and musician's dystonia}

Writer's cramp is characterized by involuntary cramping of muscles of the hand, forearm, or upper arm selectively triggered by writing. Typically, the distal muscles of the upper extremity are affected, but dystonia may progress to include more proximal muscle groups, may be triggered by other activities, and can even spread to the opposite nondominant hand. Average age of onset is in the fourth decade, and once present, symptoms rarely remit [8].

FTSD seen in musicians, a group that may be at risk due to overly practiced fine motor tasks, typically manifests in two phenotypes based on the particular instrument: musician's hand dystonia or embouchure dystonia. Musician's dystonia can occur in both amateur and professional musicians, men are affected four times as often as women, and average symptom onset is in the fourth decade [15].

Musician's hand dystonia has been reported with a variety of instruments, including piano, violin, guitar, flute, clarinet, horn and tabla, among others. Dystonia typically occurs in the hand that performs the more demanding tasks, such as the right hand in pianists and the left hand in violinists [16]. The specific pattern of abnormal muscle activation varies by instrument. For example, abnormal flexion of the fingers is typically seen in pianists and violinists, while in woodwind or brass players, extension due to lumbrical activation can occur [15]. FTSD may be exquisitely task specific, triggered by playing one instrument, but sparing the hand when a patient plays a different instrument.

Embouchure dystonia may affect brass and woodwind players, with age of onset in the fourth decade [6]. The embouchure is the critical interplay of the lips and facial muscles with the instrument's mouthpiece that controls the production of the desired air stream. Embouchure dystonias may be further classified by the pattern of abnormal movements, including embouchure tremor, involuntary lip movements and jaw closure [6].

\section{Advances in understanding}

In recent years, much effort has focused on understanding the etiology, risk factors and pathophysiology of FTSD and potential therapeutic interventions. We will review these in turn.

\section{Etiology}

The etiology of FTSD remains unknown, although recent lines of evidence suggest that both genetic and environmental factors are important [17]. Examination of family members of patients with FTSD revealed up to $25 \%$ of patients with an affected family member $[18,19]$. This is consistent with a recent study of musician's dystonia which found approximately $20 \%$ of patients with a similarly affected family member [20]. A recent genome-wide analysis has found an association with the arylsulfatase $G$ (ARSG) gene in both musician's hand dystonia and writer's cramp, but to date, a specific causative mutation within this gene has not been identified [21, 22]. Additionally, in a study of musicians with FTSD of the hand, along with patients with writer's cramp and their relatives, reduced interhemispheric inhibition as measured by transcranial magnetic stimulation (TMS) was observed in individuals where there was a positive family history for dystonia. This finding suggests that reduced interhemispheric inhibition may serve as a possible endophenotypic marker of genetic susceptibility for developing FTSD [23].

In addition to repetitive, over-practicing of a motor task, other environmental factors may contribute to the risk factors of developing FTSD. Possible risk factors include personality traits, such as perfectionism and anxiety, anatomical factors, such as hand size and joint mobility, as well as delayed onset of age of musical training $[17,24,25]$.

\section{Pathophysiology}

The pathophysiology of FTSD has been linked to abnormalities in inhibition, plasticity, and motor networks. In 1995, experiments first demonstrated decreased short intracortical inhibition (SICI) in FTSD patients compared to healthy controls using TMS [26]. Interestingly, this abnormality was found in the bilateral hemispheres of patients, despite unilateral symptoms. Recent research has therefore postulated that decreased SICI may not directly 
cause abnormal motor activation but rather facilitate the development of FTSD through other mechanisms [27, 28]. Specifically, one suggested mechanism, found in several studies of FTSD patients, is the development of impaired surround inhibition, a neural inhibitory mechanism responsible for the selective recruitment and activation of muscles necessary for a particular task with inactivation of the neighboring muscles that are unnecessary [27, 29, 30]. Consistent with the hypothesis of decreased SICI and impaired surround inhibition, multiple studies have shown a loss of dexterity and impaired independent movement of fingers of patients with either writer's cramp or musician's dystonia [31-33].

Another interesting abnormality that may contribute to FTSD pathology is maladaptive neural plasticity. While plasticity is believed to be critical to the processes of learning and memory, maladaptive neuroplastic responses in both the motor and sensory cortices have been examined in conditioning protocols using repetitive stimuli from TMS [34]. Patients with writer's cramp exhibited abnormal responses to paired associative stimulation of the median nerve and primary motor cortex. Such abnormalities included increased facilitation with spread to non-median nerve innervated muscles in addition to the absence of a typical cortical silent period [35]. More recently, experiments demonstrated a decreased short latency afferent inhibition following $1-\mathrm{Hz}$ repetitive TMS in writer's cramp, but not in normal controls [36].

Furthermore, in both musician's dystonia and writer's cramp, functional neuroimaging experiments have demonstrated abnormal cortical representations of digits and reorganization of the sensory homunculus in the sensory cortex [37-39]. Such aberrant somatotopy may be reversible with associated improved fine motor control using constraint-induced therapy [40-42]. However, another study of somatotopic mapping discovered bilateral misrepresentation of digits despite unilateral dystonic symptoms, suggesting that the disturbed somatotopy is an endophenotype for vulnerability to develop FTSD [43]. Regardless of the etiology of the aberrant somatotopy, the evidence suggests that maladaptive plasticity of FTSD impairs sensorimotor integration.

Finally, results from recent investigations have suggested a network disorder leading to FTSD, whereby involvement of the entire sensorimotor network contributes to dystonia [44]. Hyperactivation of the basal ganglia has been demonstrated in fMRI studies of writer's cramp [45]. Further aberrant basal ganglia function has been demonstrated by PET studies, which have shown decreased release of striatal dopamine during hand activation in patients with writer's cramp [46]. Cerebellar dysfunction has also been suggested to contribute to FTSD, although the specific abnormality is not well understood. Some investigations have reported increased cerebellar activity in patients with writer's cramp [47-49], while others have demonstrated decreased activity in the cerebellum during hand activation in FTSD [50, 51]. Further research is warranted to understand the precise network abnormalities; however, evidence of aberrant connections between the basal ganglia and cerebellum leading to dystonia is supported by research demonstrating that interruption of this connection leads to improvement of the dystonic symptoms [52].

\section{Treatment}

Current treatment modalities for FTSD include oral medication, chemodenervation, surgery and physical therapy. Anticholinergic agents like trihexyphenidyl, as well as other medications, such as primidone, baclofen, and phenytoin have been tried with inconsistent responses and frequent intolerable side effects [53-55]. Chemodenervation with botulinum neurotoxin (BoNT) type A has been the mainstay of treatment for FTSD.

Each of the seven known BoNT serotypes (types A-G) targets a specific SNARE protein for degradation in peripheral cholinergic neurons, thereby preventing the downstream release of acetylcholine into the neuromuscular junction. As a result, chemodenervation and subsequent muscle paralysis occur and persist for several months until the eventual degradation of BoNT and regeneration of SNARE proteins [56]. While the inhibition of acetylcholine release at the neuromuscular junction is believed to be a major component of BoNT's mechanism of action, there is increasing evidence that BoNT also acts peripherally at gamma motor neurons to reduce afferent sensory input from muscle spindles to the central nervous system and to alter sensorimotor pathways [57-61]. Treatment of limb dystonia with BoNT has demonstrated transient increased intracortical inhibition on par with normal levels of inhibition as measured by transcranial magnetic stimulation [62]. Furthermore, recent research suggests that BoNT may additionally have non-SNARE cellular targets involved in wide-ranging activities, such as cell division and apoptosis, neuritogenesis and gene expression [63].

Of the seven BoNT serotypes, only serotype A and to a lesser extent serotype B are available for clinical use, with specific formulations of each serotype characterized by different potency, immunogenicity, preparation, compound stability and heat tolerance. Notably, BoNT type B is only formally approved for the treatment of cervical dystonia, while BoNT type A has approved indications in the treatment of both neurologic and non-neurologic conditions [64]. Multiple studies have demonstrated long-lasting treatment benefits of BoNT in FTSD, but there is a delicate balance between reducing dystonic symptoms without inducing concurrent residual weakness resulting in loss of 
motor function [53, 65-68]. Even with treatment, many affected musicians are no longer able to play professionally, due to the high level of fine motor skill required for continued professional performance.

In recent years, emerging studies have investigated the role of surgery and sensorimotor retraining as therapeutic options. Thalamotomies have been performed as treatment of a variety of movement disorders since the 1950s [69]. In what was the largest published case series of patients with writer's cramp undergoing stereotactic ventro-oral thalamotomy, eleven of twelve patients reported almost complete resolution of symptoms with sustained benefit for over one year after surgery [70]. Based on its benefit for writer's cramp, stereotactic ventro-oral thalamotomy was demonstrated to improve medically refractory musician's dystonia with long-term benefit [71]. More recently in 2016, treatment with noninvasive gamma knife ventro-oral thalamotomy was shown to be effective in a case of refractory musician's dystonia for a patient who was deemed too high-risk for conventional stereotactic thalamotomy [72]. However, larger long-term follow-up studies will be necessary to evaluate the lasting efficacy of this intervention. Additionally, a small case series has investigated the role of deep brain stimulation (DBS) in the treatment of FTSD with promising results [73]. Given the invasive nature of both thalamotomies and DBS, these procedures have primarily been reserved for medically refractory cases.

Based on the idea of excessive motor excitability and aberrant sensorimotor integration in the development of FTSD, sensorimotor retraining may hold promise. Previous attempts at reducing focal dystonia symptoms by means of rehabilitation involved immobilization and splinting of the affected body part. Recent studies examining the effects of augmenting current rehabilitation techniques to include transcranial direct current stimulation have offered encouraging results. In 2014, patients with musician's dystonia displayed improvement of fine motor movements following motor retraining assisted by bi-hemispheric, noninvasive brain stimulation via transcranial direct current stimulation to the motor cortex [74]. Likewise, in 2015, transcranial direct simulation was shown to enhance the response to rehabilitation in patients with FTSD of the hand in a randomized control trial [75]. Anodal transcranial direct current stimulation targeting the cerebellum has also been shown to improve handwriting in patients with writer's cramp [76].

\section{Conclusion}

FTSD are a fascinating group of movement disorders characterized by aberrant motor overactivation during the performance of a specific, often over-practiced activity.
The triggering activity can be associated with one's occupation, leading to the disorder's further classification as an occupational dystonia. The development of such a condition can impact one's livelihood, particularly if symptoms are severe. While progress has been made in recent years in understanding the etiology, risk factors and pathophysiology of FTSD, improved therapeutic options are needed.

\section{Compliance with ethical standards}

Conflicts of interest The authors have no conflict of interest to report.

Open Access This article is distributed under the terms of the Creative Commons Attribution 4.0 International License (http://crea tivecommons.org/licenses/by/4.0/), which permits unrestricted use, distribution, and reproduction in any medium, provided you give appropriate credit to the original author(s) and the source, provide a link to the Creative Commons license, and indicate if changes were made.

\section{References}

1. Albanese A et al (2013) Phenomenology and classification of dystonia: a consensus update. Mov Disord 28(7):863-873

2. Oppenheim H (1911) Text-book of nervous diseases for physicians and students. 5th enl. and improved ed. O. Schulze \& company, Edinburgh. G. E. Stechert \& company, New York

3. Bell C (1933) Partial paralyses of the muscles of the extremities'. the nervous system of the human body. Taylor and Francis, London, pp 57-58

4. Pearce JM (2005) A note on scrivener's palsy. J Neurol Neurosurg Psychiatry 76(4):513

5. Frucht SJ (2004) Focal task-specific dystonia in musicians. Adv Neurol 94:225-230

6. Frucht SJ et al (2001) The natural history of embouchure dystonia. Mov Disord 16(5):899-906

7. Yoo SW et al (2015) Hairdresser dystonia: an unusual substantia nigra hyperechogenicity. J Neurol Sci 357(1-2):314-316

8. Sheehy MP, Marsden CD (1982) Writers' cramp-a focal dystonia. Brain 105(Pt 3):461-480

9. Defazio G, Berardelli A, Hallett M (2007) Do primary adult-onset focal dystonias share aetiological factors? Brain $130(\mathrm{Pt}$ 5):1183-1193

10. Epidemiological Study of Dystonia in Europe Collaborative, G (2000) A prevalence study of primary dystonia in eight European countries. J Neurol 247(10):787-792

11. Butler AG et al (2004) An epidemiologic survey of dystonia within the entire population of northeast England over the past nine years. Adv Neurol 94:95-99

12. Altenmuller E (2003) Focal dystonia: advances in brain imaging and understanding of fine motor control in musicians. Hand Clin 19(3):523-538 (xi)

13. Brandfonbrener A (1995) Musicians with focal dystonia: a report of 58 cases seen during a ten-year period at a performing arts medicine clinic. Med Probl Perform Artists 10:121-127

14. Greene PE, Bressman S (1998) Exteroceptive and interoceptive stimuli in dystonia. Mov Disord 13(3):549-551

15. Conti AM, Pullman S, Frucht SJ (2008) The hand that has forgotten its cunning-lessons from musicians' hand dystonia. Mov Disord 23(10):1398-1406 
16. Altenmuller E, Jabusch HC (2010) Focal dystonia in musicians: phenomenology, pathophysiology and triggering factors. Eur $\mathbf{J}$ Neurol 17(Suppl 1):31-36

17. Schmidt A et al (2013) Challenges of making music: what causes musician's dystonia? JAMA Neurol 70(11):1456-1459

18. Waddy HM et al (1991) A genetic study of idiopathic focal dystonias. Ann Neurol 29(3):320-324

19. Stojanovic M, Cvetkovic D, Kostic VS (1995) A genetic study of idiopathic focal dystonias. J Neurol 242(8):508-511

20. Schmidt A et al (2009) Etiology of musician's dystonia: familial or environmental? Neurology 72(14):1248-1254

21. Lohmann $\mathrm{K}$ et al (2014) Genome-wide association study in musician's dystonia: a risk variant at the arylsulfatase $\mathrm{G}$ locus? Mov Disord 29(7):921-927

22. Nibbeling E et al (2015) Accumulation of rare variants in the arylsulfatase G (ARSG) gene in task-specific dystonia. J Neurol 262(5):1340-1343

23. Baumer $\mathrm{T}$ et al (2016) Abnormal interhemispheric inhibition in musician's dystonia-trait or state? Parkinsonism Relat Disord 25:33-38

24. Ioannou CI, Altenmuller E (2014) Psychological characteristics in musicians dystonia: a new diagnostic classification. Neuropsychologia 61:80-88

25. Leijnse JN, Hallett M, Sonneveld GJ (2015) A multifactorial conceptual model of peripheral neuromusculoskeletal predisposing factors in task-specific focal hand dystonia in musicians: etiologic and therapeutic implications. Biol Cybern 109(1):109-123

26. Ridding MC et al (1995) Changes in the balance between motor cortical excitation and inhibition in focal, task specific dystonia. J Neurol Neurosurg Psychiatry 59(5):493-498

27. Beck S et al (2008) Short intracortical and surround inhibition are selectively reduced during movement initiation in focal hand dystonia. J Neurosci 28(41):10363-10369

28. Hallett M (2011) Neurophysiology of dystonia: the role of inhibition. Neurobiol Dis 42(2):177-184

29. Beck S, Hallett M (2011) Surround inhibition in the motor system. Exp Brain Res 210(2):165-172

30. Sohn YH, Hallett M (2004) Disturbed surround inhibition in focal hand dystonia. Ann Neurol 56(4):595-599

31. Furuya $S$ et al (2015) Losing dexterity: patterns of impaired coordination of finger movements in musician's dystonia. Sci Rep 5:13360

32. Furuya S, Altenmuller E (2013) Finger-specific loss of independent control of movements in musicians with focal dystonia. Neuroscience 247:152-163

33. Curra A et al (2004) Impairment of individual finger movements in patients with hand dystonia. Mov Disord 19(11):1351-1357

34. Siebner HR, Rothwell J (2003) Transcranial magnetic stimulation: new insights into representational cortical plasticity. Exp Brain Res 148(1):1-16

35. Quartarone A et al (2003) Abnormal associative plasticity of the human motor cortex in writer's cramp. Brain $126(\mathrm{Pt}$ 12):2586-2596

36. Baumer T et al (2007) Abnormal plasticity of the sensorimotor cortex to slow repetitive transcranial magnetic stimulation in patients with writer's cramp. Mov Disord 22(1):81-90

37. Elbert $\mathrm{T}$ et al (1998) Alteration of digital representations in somatosensory cortex in focal hand dystonia. Neuroreport 9(16):3571-3575

38. Nelson AJ, Blake DT, Chen R (2009) Digit-specific aberrations in the primary somatosensory cortex in Writer's cramp. Ann Neurol 66(2):146-154

39. Bara-Jimenez W et al (1998) Abnormal somatosensory homunculus in dystonia of the hand. Ann Neurol 44(5):828-831
40. Candia V et al (2003) Effective behavioral treatment of focal hand dystonia in musicians alters somatosensory cortical organization. Proc Natl Acad Sci USA 100(13):7942-7946

41. Candia V et al (2002) Sensory motor retuning: a behavioral treatment for focal hand dystonia of pianists and guitarists. Arch Phys Med Rehabil 83(10):1342-1348

42. Candia V et al (1999) Constraint-induced movement therapy for focal hand dystonia in musicians. Lancet 353(9146):42

43. Meunier $\mathrm{S}$ et al (2001) Human brain mapping in dystonia reveals both endophenotypic traits and adaptive reorganization. Ann Neurol 50(4):521-527

44. Furuya S, Hanakawa T (2016) The curse of motor expertise: usedependent focal dystonia as a manifestation of maladaptive changes in body representation. Neurosci Res 104:112-119

45. Blood AJ et al (2004) Basal ganglia activity remains elevated after movement in focal hand dystonia. Ann Neurol 55(5):744-748

46. Berman BD et al (2013) Striatal dopaminergic dysfunction at rest and during task performance in writer's cramp. Brain $136(\mathrm{Pt}$ 12):3645-3658

47. Odergren T, Stone-Elander S, Ingvar M (1998) Cerebral and cerebellar activation in correlation to the action-induced dystonia in writer's cramp. Mov Disord 13(3):497-508

48. Preibisch $\mathrm{C}$ et al (2001) Cerebral activation patterns in patients with writer's cramp: a functional magnetic resonance imaging study. J Neurol 248(1):10-17

49. Lerner A et al (2004) Regional cerebral blood flow correlates of the severity of writer's cramp symptoms. Neuroimage 21(3):904-913

50. Kadota $\mathrm{H}$ et al (2010) An fMRI study of musicians with focal dystonia during tapping tasks. J Neurol 257(7):1092-1098

51. Moore RD et al (2012) Individuated finger control in focal hand dystonia: an fMRI study. Neuroimage 61(4):823-831

52. Chen $\mathrm{CH}$ et al (2014) Short latency cerebellar modulation of the basal ganglia. Nat Neurosci 17(12):1767-1775

53. Jabusch HC et al (2005) Focal dystonia in musicians: treatment strategies and long-term outcome in 144 patients. Mov Disord 20(12):1623-1626

54. Jankovic J (2006) Treatment of dystonia. Lancet Neurol 5(10):864-872

55. Balash Y, Giladi N (2004) Efficacy of pharmacological treatment of dystonia: evidence-based review including meta-analysis of the effect of botulinum toxin and other cure options. Eur J Neurol 11(6):361-370

56. Simpson LL (2004) Identification of the major steps in botulinum toxin action. Annu Rev Pharmacol Toxicol 44:167-193

57. Rosales RL, Dressler D (2010) On muscle spindles, dystonia and botulinum toxin. Eur J Neurol 17(Suppl 1):71-80

58. Rosales RL et al (1996) Extrafusal and intrafusal muscle effects in experimental botulinum toxin-A injection. Muscle Nerve 19(4):488-496

59. Palomar FJ, Mir P (2012) Neurophysiological changes after intramuscular injection of botulinum toxin. Clin Neurophysiol 123(1):54-60

60. Matak I, Lackovic Z (2014) Botulinum toxin A, brain and pain. Prog Neurobiol 119-120:39-59

61. Giladi N (1997) The mechanism of action of botulinum toxin type $\mathrm{A}$ in focal dystonia is most probably through its dual effect on efferent (motor) and afferent pathways at the injected site. J Neurol Sci 152(2):132-135

62. Gilio F et al (2000) Effects of botulinum toxin type A on intracortical inhibition in patients with dystonia. Ann Neurol 48(1):20-26

63. Matak I, Lackovic Z (2015) Botulinum neurotoxin type A: actions beyond SNAP-25? Toxicology 335:79-84 
64. Bentivoglio AR et al (2015) Clinical differences between botulinum neurotoxin type A and B. Toxicon 107(Pt A):77-84

65. Hallett $\mathrm{M}$ et al (2013) Evidence-based review and assessment of botulinum neurotoxin for the treatment of movement disorders. Toxicon 67:94-114

66. Lungu $\mathrm{C}$ et al (2011) Long-term follow-up of botulinum toxin therapy for focal hand dystonia: outcome at 10 years or more. Mov Disord 26(4):750-753

67. Mejia NI, Vuong KD, Jankovic J (2005) Long-term botulinum toxin efficacy, safety, and immunogenicity. Mov Disord 20(5):592-597

68. Schuele $\mathrm{S}$ et al (2005) Botulinum toxin injections in the treatment of musician's dystonia. Neurology 64(2):341-343

69. Andrew J, Fowler CJ, Harrison MJ (1983) Stereotaxic thalamotomy in 55 cases of dystonia. Brain 106(Pt 4):981-1000

70. Taira T, Hori T (2003) Stereotactic ventrooralis thalamotomy for task-specific focal hand dystonia (writer's cramp). Stereotact Funct Neurosurg 80(1-4):88-91
71. Horisawa S et al (2013) Long-term improvement of musician's dystonia after stereotactic ventro-oral thalamotomy. Ann Neurol 74(5):648-654

72. Horisawa $S$ et al (2016) Gamma knife ventro-oral thalamotomy for musician's dystonia. Mov Disord

73. Fukaya $\mathrm{C}$ et al (2007) Thalamic deep brain stimulation for writer's cramp. J Neurosurg 107(5):977-982

74. Furuya $S$ et al (2014) Surmounting retraining limits in musicians' dystonia by transcranial stimulation. Ann Neurol 75(5):700-707

75. Rosset-Llobet J, Fabregas-Molas S, Pascual-Leone A (2015) Effect of transcranial direct current stimulation on neurorehabilitation of task-specific dystonia: a double-blind, randomized clinical trial. Med Probl Perform Artists 30(3):178-184

76. Bradnam LV et al (2015) Anodal transcranial direct current stimulation to the cerebellum improves handwriting and cyclic drawing kinematics in focal hand dystonia. Front Hum Neurosci 9:286 